\title{
The demography of human development and climate change vulnerability: A projection exercise
}

\author{
Jesús Crespo Cuaresma and Wolfgang Lutz*
}

\begin{abstract}
We propose a methodological framework aimed at obtaining projections of the Human Development Index (HDI) that can be used to assess the degree of vulnerability of future societies to extreme climatic events. By combining recent developments in the modeling and projection of population by age, sex, and educational attainment, our modeling set-up ensures that the different components of the HDI are projected using a self-contained, consistent modeling effort. We develop scenarios that correspond to the Shared Socioeconomic Pathways (SSPs) developed in the context of the Intergovernmental Panel on Climate Change (IPCC), and thus present a projection framework that can be used to expand the evaluation of the potential mitigation and adaptation challenges associated with climate change in general, and with vulnerability to natural disasters in particular.
\end{abstract}

\section{Introduction}

To estimate the degree to which future societies will be vulnerable to climate change, we must assess several dimensions of human development, and construct quantitative scenarios that include potential changes in income, education, and health at the global level over the coming decades. The development of socioeconomic scenarios plays a central role in research dealing with the quantification of climate change impacts, as well as in the design of policy

\footnotetext{
* Jesús Crespo Cuaresma (corresponding author), Wittgenstein Centre for Demography and Global Human Capital (IIASA, VID/ÖAW, WU), Vienna University of Economics and Business (WU), Austrian Institute of Economic Research (WIFO), International Institute for Applied Systems Analysis (IIASA), Vienna, Austria

Email: Jesus.Crespo.Cuaresma@wu.ac.at

Wolfgang Lutz, Wittgenstein Centre for Demography and Global Human Capital (IIASA, VID/ÖAW, WU), International Institute for Applied Systems Analysis (IIASA), Vienna Institute of Demography, Austrian Academy of Sciences, Vienna University of Economics and Business (WU), Vienna, Austria
} 
responses in the framework of the Intergovernmental Panel on Climate Change (IPCC) 5th Assessment Report on climate change (see for example Kriegler et al. 2012). Investigating the human dimension of resilience associated with future climate extremes and the corresponding higher risks of (climatic) natural disasters complements the study of the physical dimension of such risks.

In its approaches to dealing with climate change, the international community has been gradually moving away from an almost exclusive focus on mitigation, and toward the recognition of the need to prepare for adapting to changes in the climate that are already unavoidable (see, for example, IPCC 2014). In making this shift, policy-makers and scholars are also increasingly acknowledging that when trying to determine which populations are most vulnerable to climate change, we need to take into account not just where people are, but also who they are in terms of their demographic characteristics and capabilities. A recent international scientific panel on Population and Sustainable Development highlighted this issue in its published action points: "(i) Recognize that the numbers, characteristics, and behaviors of people are at the heart of sustainable development challenges and of their solutions. (ii) Identify subpopulations that contribute most to environmental degradation and those that are most vulnerable to its consequences. In poor countries especially, these subpopulations are readily identifiable according to age, gender, level of education, place of residence, and standard of living." (Lutz et al. 2014).

This focus on relative vulnerability is rather recent among researchers who deal with natural disasters and adaptation, as their primary focus has traditionally been on location. The importance of demographic factors - and particularly of the changing educational composition of the population-in human survival and wellbeing as the climate changes has recently been investigated in great detail (see Butz et al. 2014). Education has been shown to be a way for individuals to acquire knowledge, skills, and competencies that can directly or indirectly influence their adaptive capacity; and thus reduce risk. There have been several recent studies on this topic, including an individual-level study of disaster preparedness during the 2012 Indian Ocean earthquakes among households located along the Andaman coast in the Phang Nga province. The study found that formal education-measured at the individual, household, and community levels-increased the likelihood that households had taken preparedness actions (Muttarak and Pothisiri 2013). While having been affected by the 2004 tsunami was clearly associated with increased emergency preparedness, education was also a factor in determining whether households anticipated the risk and took preparedness actions, even among those who lacked disaster experience.

In another study, Frankenberg et al. (2013) used longitudinal survey data collected in two provinces on the island of Sumatra, Indonesia, before and after the 2004 Indian Ocean tsunami to examine the extent to which education confers protection against natural disaster at the individual level. They found that education clearly affected the ability of individuals to cope with the disaster over the longer term, as the better educated individuals were in better psycho-social health five years after the tsunami. These individuals were less likely than others to have been living under 
precarious conditions, and appear to have been more adept at compensating for the loss of income following the tsunami.

Similar evidence on the association between education and vulnerability has been reported at the community level. Using comprehensive village-level data in Nepal, KC (2013) found strong effects of education on the extent to which villages hit by floods and landslides lost human and animal lives and suffered other forms of household damage. After comparing the effect of education with those of income and wealth, the author concluded that education had a stronger and more consistent effect on the level of damage due to floods and landslides in Nepal. Likewise, Pichler and Striessnig (2013) used data from qualitative interviews conducted in Cuba and the Dominican Republic to compare the disaster vulnerability of these two island states. Even though the two countries are fairly similar in terms of the extent of their exposure to extreme natural events, their disaster outcomes differ greatly. The Cuban population is one of the most educated in the developing world, and their responses to disaster tend to be highly effective. By contrast, the interviews strongly confirmed that the lack of education and of literacy in the Dominican Republic makes the population more vulnerable, as many people have difficulties even understanding warnings about upcoming danger.

Using national-level time series of disaster fatalities around the world, a study by Striessnig et al. (2013) found significant evidence that education plays a role in reducing disaster fatalities. However, they found no evidence confirming the widespread assumption that income per capita is associated with reduced vulnerability after controlling for other key determinants of socioeconomic development, as well as for exposure to risk. They also studied disaster deaths using the Human Development Index (HDI) and its three constituent components. While the aggregate HDI was shown to be strongly correlated with the disaster vulnerability of each country, education was found to be the most significant of the individual components. The results presented in Patt et al. (2010) reinforce the view that the information contained in the HDI is well suited to serve as a disaster vulnerability indicator. In their work, Patt et al. (2010) found that countries that have a relatively high ranking on the HDI, and have thus made improvements in human development that have been captured in the HDI, tend to be less vulnerable to climatic risks. Life expectancy (as a proxy for health) and educational attainment levels have often been proposed as important determinants of vulnerability and adaptive capacity (see Brooks et al. 2005). Income, as a determinant of social vulnerability, has been found to ameliorate the impacts of extreme climate events (see, for instance, Adger 1999). The existing studies that have addressed the importance of the three components of the HDI as predictors of vulnerability have tended to emphasize the need to approach this issue in a comprehensive manner.

The insights provided by such robust results imply that examining the dynamics of income, education attainment, and life expectancy can provide us with very valuable information about the extent to which specific populations are vulnerable to future natural disasters. In particular, linking the projections of the HDI to the climate change scenarios used for the assessment of climatic disaster risks should 
generate a powerful source of information for policy-makers. In this contribution, we combine a set of recent methodologies that are capable of doing precisely that. The framework utilizes recent developments in population and income projection methods (see KC and Lutz 2015 and Crespo Cuaresma 2015, respectively) in order to obtain projected paths for the Life Expectancy Index, the Education Index and the Income Index that compose the HDI; and, thus, for the HDI itself.

The studies reviewed above have explicitly investigated differential vulnerability to recently observed natural disasters, which is not the same as vulnerability to future climate change. However, there are reasons to assume that disaster vulnerability is generally isomorphic to the vulnerability that will result from certain aspects of future climate change, and particularly from the projected higher incidence of extreme events (see IPCC, 2013). It therefore seems highly relevant to project the HDI and its three components into the future using a range of scenarios. Such a projection can provide us with a quantitative handle for assessing future differential vulnerability to climate change. Following KC and Lutz (2015), we use methods of multi-dimensional population dynamics to obtain projections of human capital for all countries of the world. We measure human capital by examining the characteristics of the population, including age, sex, and educational attainment. The importance of human capital as a fundamental driver of changes in income per capita has been stressed in the theoretical and the empirical economic growth literature. Starting with the effects of education on labor productivity, which is a robust empirical stylized fact at the microeconomic level, several recent contributions have recognized the role played by the stock of human capital (in addition to the rate of accumulation) as a catalyst of technological innovation and of foreign technology adoption (see Nelson and Phelps 1966 and Benhabib and Spiegel 1994). The availability of new global data on populations by age, sex, and educational attainment (see Lutz et al. 2007) has enabled researchers to investigate the effect on economic growth of the distribution of education across age groups. The results in Lutz et al. (2008) and Crespo Cuaresma and Mishra (2011) show that differences in the growth of income across countries and over time can be better predicted if the age dimension of human capital is incorporated into the modeling framework.

In the spirit of such modeling strategies, we complement the life expectancy and educational attainment projections that constitute the input to the Life Expectancy Index and the Education Index with income projections obtained using econometric models for per capita income growth in the spirit of Crespo Cuaresma (2015). Such econometric specifications account for the dual role of improvements in human capital as a determinant of labor productivity and of the ability of countries to make new technological advancements and to adopt foreign technologies. Projections of income per capita can be obtained by combining specifications that are estimated using a panel dataset of historical data with calibrations of the underlying parameters. This approach allows us to place the underlying storylines corresponding to the different scenarios in the context of global convergence trends and overall total factor productivity growth. This modeling set-up ensures that 
the different components of the HDI are projected using an internally consistent modeling structure. Our method further ensures that the components are comparable with the storylines that frame the scenarios put forward by Kriegler et al. (2012), and that are used as Shared Socioeconomic Pathways in the IPCC's 5th Assessment Report. Thus, we are able to tie the discussion on the human dimension of vulnerability to catastrophic risks to standardized climate change scenarios.

The set of HDI projections presented in the paper represents a unique, selfcontained, and coherent modeling exercise, which combines a set of specifications that deliver internally consistent predictions of the different components of the index. The modeling framework builds upon a series of modern contributions to the literature of demographic projections aimed at informing climate change research (see KC and Lutz 2015).

The paper is structured as follows. The methods used to obtain the HDI projections are presented in Section 2. In Section 3 the characteristics of the HDI projections up to the year 2075 are described, and the different scenarios corresponding to the IPCC's storylines are compared. Section 4 concludes.

\section{A methodological framework for human development index projections}

\subsection{The human development index}

Following the changes introduced in the United Nations Development Programme (2013), the HDI has been computed as the geometric mean of three indicators designed to capture the income, health, and education dimensions of human development. ${ }^{1}$ The income index is a function of gross national income (GNI) per capita; the health index is based on life expectancy at birth; and the education index is a geometric average of actual and expected educational attainment, as measured by years of schooling. Analytically, the HDI for year $t$ in a given country is thus given by

$$
H D I_{t}=\sqrt[3]{I_{Y, t} I_{L E, t} I_{E D, t}},
$$

where $I_{Y, t}, I_{L E, t}$ and $I_{E D, t}$ denote the indices for income, life expectancy, and education, respectively. The index for income is given by

$$
I_{Y, t}=\frac{\ln Y_{t}-\ln Y_{M I N}}{\ln Y_{M A X}-\ln Y_{M I N}},
$$

where $Y_{t}$ is GNI per capita, while $Y_{M A X}$ and $Y_{M I N}$ are predetermined maximum and minimum historical values for income. The HDI 2010 uses $\$ 108,211$ and $\$ 163$ (at 2005 PPP-adjusted prices) for $Y_{M A X}$ and $Y_{M I N}$, respectively.

1 See Klugman et al. (2011) for a detailed account of the construction of the 2010 version of the HDI, as well as its limitations. 
The life expectancy index, $I_{L E, t}$ takes a form similar to that of $I_{Y, t}$, albeit without a logarithmic transformation of the underlying variable,

$$
I_{L E, t}=\frac{L E_{t}-L E_{M I N}}{L E_{M A X}-L E_{M I N}},
$$

where $L E, t$ denotes life expectancy at birth and the maximum and minimum values $\left(L E_{M A X}-L E_{M I N}\right)$ are set to 83.2 and 20 , respectively, following UNDP (2010).

The education dimension of the human development index is in turn constructed as the arithmetic mean of two indicators: mean years of schooling (divided by a measure of maximum years of schooling) and expected years of schooling (divided by a measure of maximum expected years of schooling),

$$
I_{E D, t}=\frac{1}{2} I_{M Y S, t}+\frac{1}{2} I_{E Y S, t}=\frac{1}{2} \frac{M Y S_{t}}{M Y S_{M A X}}+\frac{1}{2} \frac{E Y S_{t}}{E Y S_{M A X}} .
$$

In the expression given by equation (4), $M Y S_{t}$ stands for the mean years of schooling of an individual aged 25 or older, while $E Y S_{t}$ measures the years of schooling a child is expected to attain given current enrolment rates. Based on historical data obtained from UNDP (2010), MYS $S_{M A X}$ and $E Y S_{M A X}$ are set to 13.2 and 20.6 years, respectively.

The way the HDI combines the indices that measure the progress made on these three dimensions of human development has not been free of criticism. Klugman et al. (2011) presented some of the controversies related to the aggregation of the three components, while Ravallion (2010) and Ravallion (2011) highlighted some problems related to the existing trade-offs between the dimensions that compose the HDI. A potential solution to the deficiencies described by Ravallion (2010), Ravallion (2011) and Chakravarty (2011) is the aggregation of the individual income, life expectancy, and education indices using the generalized HDI in Chakravarty (2003). ${ }^{2}$ Since the projection framework presented in our contribution is based on extrapolating the dynamics of each of the components of the HDI individually, different aggregation methods can be used within this framework. While we acknowledge the theoretical controversies surrounding the aggregation methods used in the HDI, we present projections based on the current HDI aggregation methodology in order to ensure comparability with existing studies.

\subsection{Projecting life expectancy}

A model used to project life expectancy in all countries of the world has recently been applied in the new Wittgenstein Centre scenarios for population and human capital in the 21 st century. This model, which has been extensively documented in Lutz et al. (2014), is based on the broad assumption of long-term global convergence.

2 Some of these criticisms are assessed by Klugman et al. (2011). 
There are two main concepts of convergence in the literature. The first, betaconvergence, occurs when the growth rate of the variable of interest (normally the growth rate in GDP) depends negatively on its prior value. Controlling for the influence of other factors, this produces conditional convergence, whereby the level of convergence depends on those other factors. The second concept is sigmaconvergence, which occurs when the dispersion of the indicator decreases. Using the concept of sigma-convergence in absolute terms, the model produces female life expectancy forecasts for all countries. Exceptions are made for countries affected by HIV-AIDS. Due to the specific dynamics of HIV-AIDS-related mortality, the UN Medium Variant assumptions based on a model by UNAIDS are used for those countries until 2050, after which the models and the rules of convergence are applied (see KC et al. 2014). In general, this convergence approach is based on the claim that "national mortality trends should be viewed in a larger international context rather than being analysed and projected individually" (Lee, 2003). The model also follows the argument made by Torri and Vaupel (2012) that life expectancy in different countries tends to be positively correlated, and that the life expectancies of particular countries can be projected by forecasting the best-practice level, and then the gap between the national performance and the best-practice level.

In the model used for the projections (Garbero and Sanderson 2014), Japan is identified as the current global forerunner in female life expectancy. Under the medium scenario, the life expectancy at birth of a Japanese woman is assumed to grow two years per decade, from 86.1 years in 2005-2010 to 104.2 in 20952100. Next, we identify the regional forerunners ( 22 regions) for which female life expectancy is projected to converge with that of Japan, and is thus expected to grow two years per decade. After the life expectancies for the regional forerunners are projected using the estimates of a dynamic panel data model, a similar specification is applied to the countries within each region that are assumed to follow their regional forerunners. The advantage of this convergence model is that it is based on empirical data. In addition, it takes into account the heterogeneous country-specific historical experiences, as well as the differences in the gains made by forerunners and laggards over time and across regions. Thus, the model takes into account the structural as well as the stochastic components that contribute to life expectancy trends over time, and is therefore able to generate unbiased parameters upon which the new forecasts can be based. These model-based results are then blended with the country-specific expert assessments, as detailed in Garbero and Pamuk (2014).

Once the medium overall life expectancy at birth trajectories for five-year periods for 2010-2100 have been defined for women in 196 countries, the life expectancy trajectories for males can be derived by applying the difference between the female and the overall life expectancy in the UN medium variant (United Nations, 2011). Next, gender-specific education differentials in mortality are introduced as differences in life expectancy at age 15. Based on current empirical data, the life expectancy gap at age 15 between the populations with no education and with tertiary education is assumed to be six years for men and four years for women. This is operationalized by assuming two years' difference between 'completed 
primary' and 'completed lower secondary', and one year for the remaining levels of attainment. Finally, for children up to age 15 the differential mortality is introduced through the mother's education. Here the differentials in terms of the relative ratio of mortality rates for individuals in the completed upper secondary category are 1.8, $1.7,1.6,1.4,1.0$, and 0.8 ; in ascending order of educational attainment. These values are based on the averages of under-five mortality rates in the countries included in the Demographic and Health Survey (DHS) program. ${ }^{3}$

These procedures result in a full set of age-, sex-, and education-specific mortality trajectories for all of the countries for the medium scenario. The high and low scenarios follow the same logic, with the main difference being that the life expectancy gains experienced by the global forerunner (Japan) are assumed to be three years per decade in the high scenario, and one year per decade in the low scenario. Some more specific assumptions are made for the nearer term in some high mortality countries, as detailed in KC et al. (2014).

\subsection{Projecting educational attainment}

The education projections used here are identical to those used in the recent contribution by Lutz et al. (2014). Unlike other existing population projection exercises, they explicitly account for the systematic differences in fertility, mortality, and migration by educational attainment level. They start with empirical data on educational attainment distributions for seven educational categories by age and sex for all countries around 2010. This empirical database is described in detail in KC et al. (2014). The medium education scenario is the so-called Global Education Trend (GET) scenario. It is based on a Bayesian model that estimates the most likely future trajectory in education-specific progression rates to higher levels using the cumulative experience of all countries over the past 40 years.

More specifically, the proportional distribution by six levels of educational attainment in the age group 30-34 is first extracted from the Bayesian model as a median trajectory of thousands of iterations (Barakat and Durham, 2014). It represents the final education distribution of a particular cohort, which, apart from changes due to education differentials in mortality and migration, may be expected to remain unchanged over the cohort's remaining life span. In order to calculate the education distribution under age 30 , the education-specific proportions in the age group 30-34 are back-casted to ages 15-19, 20-24, and 25-29; such that attainment in the younger age groups follows the country-specific experience in the past. We impose convergence in cases in which attainment progressions in certain education groups are occurring at later ages. For example, we expect that primary education, which typically lasts four years, will be completed by age 15 . These sets

3 See http://dhsprogram.com/where-we-work/country-list.cfm. 
of education distributions are prepared for each sex separately, and for all periods from 2010 to 2100 .

In addition to the medium GET scenario, three alternative scenarios are defined. These scenarios are used to explore the sensitivity of the population projections to our education assumption. The results suggest that altering education can result in differences in the projected population of almost one billion by 2060. The three scenarios are defined as follows:

- Fast Benchmark or Fast Track (FT). In this scenario, the most rapid countryspecific expansion parameters are applied to all countries throughout the projection period. In other words, all countries follow the educational development paths taken in the past by the frontrunners in east and southeast Asia.

- Constant Enrolment Rates (CER). For this scenario, the attainment shares at age 30-34 of future cohorts are fixed at the levels observed in the base year (but are adjusted if younger age groups in the base year are already exhibiting attainment levels that are higher than predicted).

- Constant Enrolment Numbers (CEN). This scenario differs from CER, as country-specific attainment by age (under 35) and sex is kept constant at the absolute levels observed in the base year. While CER is a pessimistic low scenario, CEN could be either lower than CER for countries with larger younger cohorts, or higher than CER in countries with smaller younger cohorts. This scenario is of particular interest when considering the consequences of keeping levels of investment in schools and universities constant in the coming decades.

The frequently used indicator of mean years of schooling (MYS) has the advantage of expressing the entire distribution of educational attainment in a single number. It is therefore often used in cross-country comparisons as well as in models (e.g. economic and environmental models) as the unique indicator of educational attainment and human capital stock. The computation of mean years of schooling from a given educational attainment distribution is complex for two main reasons. First, the standard duration of different levels of schooling can vary from country to country; and within countries school levels can vary in length depending on the particular course of study (e.g. general secondary or vocational secondary). Second, the calculation is biased by the share of students who do not complete the full course of any level, which can be substantial in some countries. To address these difficulties, the methodology used here computes mean years of schooling as the weighted mean of six educational levels (and zero for no education). This procedure, as described in KC et al. (2014), takes into account country-specific educational systems, as well as changes in these systems over time. Information on the duration of schooling for each completed ISCED level is taken from the UNESCO Institute for Statistics 
(UNESCO UIS) database ${ }^{4}$ For the cohorts who attended school prior to 1970 , the last year for which UIS provides information, we assume durations identical to those from the last year of observation. For the projected periods, we use the constant durations given for 2010 .

For the countries and the cohorts with nearly universal primary education, we find that incomplete primary has a longer duration among the fraction of individuals who dropped out of primary school. This relationship holds for both genders and across broad world regions, with the exception of south Asia. For the regions where the data needed for such models were not available-like Europe, North America, Australia, and Oceania, as well as the former Soviet republics in central Asia-we assume the same relationship as in Latin America. Thus, we assume that the duration of schooling is long for those individuals with an incomplete primary education, since these regions generally have high levels of educational attainment. In these regions the fraction of individuals in the incomplete primary education category is negligible overall, even among older cohorts.

\subsection{Projecting income per capita}

The modeling context by Crespo Cuaresma (2015) provides an internally consistent framework to obtain GDP projections using human capital trajectory scenarios. Starting with an aggregate production function with heterogeneous labor input defined by age and educational attainment groups, Crespo Cuaresma (2015) proposes the integration of population projections (by age and educational attainment) to obtain projected income paths that can be used for the assessment of climate change impacts.

In addition to taking into account the labor productivity effects of education, in the spirit of Benhabib and Spiegel (1994) the aggregate production includes the impact of educational improvements on technological progress, in the form of both innovation and technology adoption. These effects are specified by including the interaction of income per capita and educational attainment (by age groups) as determinants of GDP growth. This specification is then estimated to obtain elasticities for the projection exercise. The starting point of the income projection exercise is thus given by the aggregate production function

$$
Y_{i t}=A_{i t} K_{i t}^{\alpha} \prod_{j=0}^{3} \prod_{k=1}^{2} L_{i, j k t}^{\beta_{j k}}
$$

where $Y_{i t}$ is total GDP in country $i$ at time $t, A_{i t}$ is total factor productivity (TFP), $K_{i t}$ is the capital stock, and $L_{i, j k t}$ corresponds to the labor input in age group $k$ ( $k=1,2$ denoting the younger and older age group) with educational attainment $j$

4 See http://www.uis.unesco.org/datacentre/. 
(from $j=0$ - no education - to $j=3$ - some tertiary education level attained). In logs and taking first differences, such a production function can be written as

$$
\Delta \log Y_{i t}=\Delta \log A_{i t}+\alpha \Delta \log K_{i t}+\sum_{j=0}^{3} \sum_{k=1}^{2} \beta_{j k} \Delta \log L_{i, j k t}
$$

Assuming that the growth rate of TFP is affected by the stock of human capital, both directly and through its interaction with income per capita, results in the following specification

$$
\begin{aligned}
\Delta \log Y_{i t}= & \alpha \Delta \log K_{i t}+\sum_{j=0}^{3} \sum_{k=1}^{2} \beta_{j k} \Delta \log L_{i, j k t}+\delta \log \frac{Y_{i t}}{L_{i t}}+ \\
& +\sum_{j=1}^{3} \theta_{j} \sum_{k=1}^{2} \frac{L_{i, j k t}}{L_{i t}}+\sum_{j=1}^{3} \phi_{j} \log \frac{Y_{i t}}{L_{i t}} \sum_{k=1}^{2} \frac{L_{i, j k t}}{L_{i t}}
\end{aligned}
$$

This is the model that is actually used to estimate the parameters for the projection model based on a global dataset for the period 1970-2005 (at five-year nonoverlapping intervals) (see Crespo Cuaresma (2015)). The panel structure of the dataset allows for the estimation of fixed effects models, and thus accounts for unobservable differences in income growth across countries that have remained constant in the sample period; as well as for common GDP growth shocks that affect all countries. Using projections of population by age and education, together with assumptions about the accumulation path of physical capital, global income convergence patterns, and technology growth; paths of income per capita that are consistent with the narratives used in the assessment of the impacts of climate change are obtained for 154 countries.

\section{HDI projections: Shared socioeconomic pathways and climate change vulnerability}

The Intergovernmental Panel on Climate Change (IPCC) has recently finalized its Fifth Assessment Report (AR5). In this context, members of the global community who model the Integrated Assessment (IA) and Vulnerability, Risk, and Adaptation (VRA) have agreed to refer to a new common set of Shared Socioeconomic Pathways (SSPs) that describe a range of future scenarios, with a focus on the social and economic mitigation and adaptation challenges. Unlike the previous generation of SRES scenarios (Nakicenovic et al. 2000), which considered total population size and total GDP to be the only relevant socioeconomic factors, and which essentially reduced population to a scaling factor for the denominator of different variables; this new set of scenarios provides much richer socioeconomic content, including a range of population scenarios by age, sex, and six levels of educational attainment 
for all countries in the world. The main reason for moving to much more detailed characterizations of the socioeconomic aspects of global change is that the SSPs are no longer primarily designed to describe the factors contributing to $\mathrm{CO}_{2}$ emissions (the challenges for mitigation), but rather to explore the extent to which societies are vulnerable to or are able to adapt to climate change. Demographic dimensionssuch as age, sex, level of education, and urbanization - are considered key factors that should be explicitly included in the scenarios.

The SSPs were designed in a lengthy process involving most of the leading global change modeling teams. The process was guided by the goal of providing a comprehensive description of the socioeconomic challenges that may be expected to arise in conjunction with climate change mitigation and adaptation in the future. In addition to analyzing trends in population, education, and urbanization; the scenarios also covered several dimensions of the economy, particularly energy consumption and the carbon intensity of alternative technologies that might be developed in the future. A summary of the five SSP scenarios is provided in Table 1, and the characteristics of each scenario are presented below.

- SSP1 (Sustainability - Rapid social development): This scenario assumes a future in which the world is moving toward a more sustainable path with a relatively low global population, as educational and health investments accelerate the demographic transition. There are major improvements in human capital, while the fertility rate in the OECD countries is moderately high.

- SSP2 (Continuation - Medium Social Development): This is the middle-ofthe-road scenario in which trends typical of recent decades continue, with slow progress being made toward achieving development goals, reducing resource and energy intensity, and decreasing fossil fuel dependency. In demographic terms, this scenario is identical to the medium scenario in the new global human capital projections produced by the Wittgenstein Centre for Demography and Global Human Capital (Lutz et al. 2014).

- SSP3 (Fragmentation - Stalled Social Development): In this scenario the world is separated into regions characterized by extreme poverty and pockets of moderate wealth, and large numbers of countries are struggling to maintain living standards for rapidly growing populations. The demographic transition is stalled in countries that still have high fertility, as educational levels in these countries remain low.

- In addition, SSP4 (Inequality) and SSP5 (Conventional Development) describe pathways in which large segments of the population remain vulnerable (high adaptation and low mitigation challenges), and are stuck in a pattern of carbon-intensive conventional economic growth (high mitigation and low adaptation challenges).

Using the methods described in the previous section, we obtain projection paths for each of the indices that compose the HDI, and aggregate them to create projections of the HDI. This is done for 154 countries for which data 
Table 1:

Shared socio economic pathways: Assumptions

\begin{tabular}{llllll}
\hline & Country groupings & Fertility & Mortality & Migration & Education \\
\hline SSP1 & HiFert & Low & Low & Medium & High (FT-GET) \\
& LoFert & Low & Low & Medium & High (FT-GET) \\
& Rich-OECD & Medium & Low & Medium & High (FT-GET) \\
SSP2 & HiFert & Medium & Medium & Medium & Medium (GET) \\
& LoFert & Medium & Medium & Medium & Medium (GET) \\
& Rich-OECD & Medium & Medium & Medium & Medium (GET) \\
SSP3 & HiFert & High & High & Low & Low (CER) \\
& LoFert & High & High & Low & Low (CER) \\
& Rich-OECD & Low & High & Low & Low (CER) \\
SSP4 & HiFert & High & High & Medium & CER-10\%/GET \\
& LoFert & Low & Medium & Medium & CER-10\%/GET \\
& Rich-OECD & Low & Medium & Medium & CER/CER-20\% \\
SSP5 & HiFert & Low & Low & High & High (FT-GET) \\
& LoFert & Low & Low & High & High (FT-GET) \\
& Rich-OECD & High & Low & High & High (FT-GET) \\
\hline
\end{tabular}

are available (see the appendix). We accommodate the changes in the maximum value of life expectancy, income, and years of schooling over the projection period by substituting $Y_{M A X}, L E_{M A X}, M Y S_{M A X}$ and $E Y S_{M A X}$ with the corresponding maximum value across economies, projection years, and scenarios. Breaking with the standard practice for computing the HDI, we define the maximum reference point over the full projection period instead of redefining it by year. Using this approach, we are able to create a set of projections that are comparable across countries and scenarios, although we lose comparability with past values of the HDI. It should be noted, however, that other standardization methods could have been used. As projections of income per capita, years of schooling, and life expectancy are available, new human development indicators that may be relevant for policy could be created.

Figure 1 presents the distribution of the HDI and its components across countries for the five SSP scenarios over the period $2010-2075 .^{5}$ In a world in which development is polarized, as projected in SSP3 and SSP4, the HDI values are highly dispersed over the whole projection horizon, for both the aggregate index and the income, life expectancy, and education sub-indices. In SSP1 and SSP5, by contrast, the HDI levels converge at high levels, with a small degree of dispersion occurring at the end of the projection horizon. These patterns of convergence can be observed in

5 The projected HDI series can be found at http://www.iiasa.ac.at/web/home/research/researchPrograms/WorldPopulation/. 


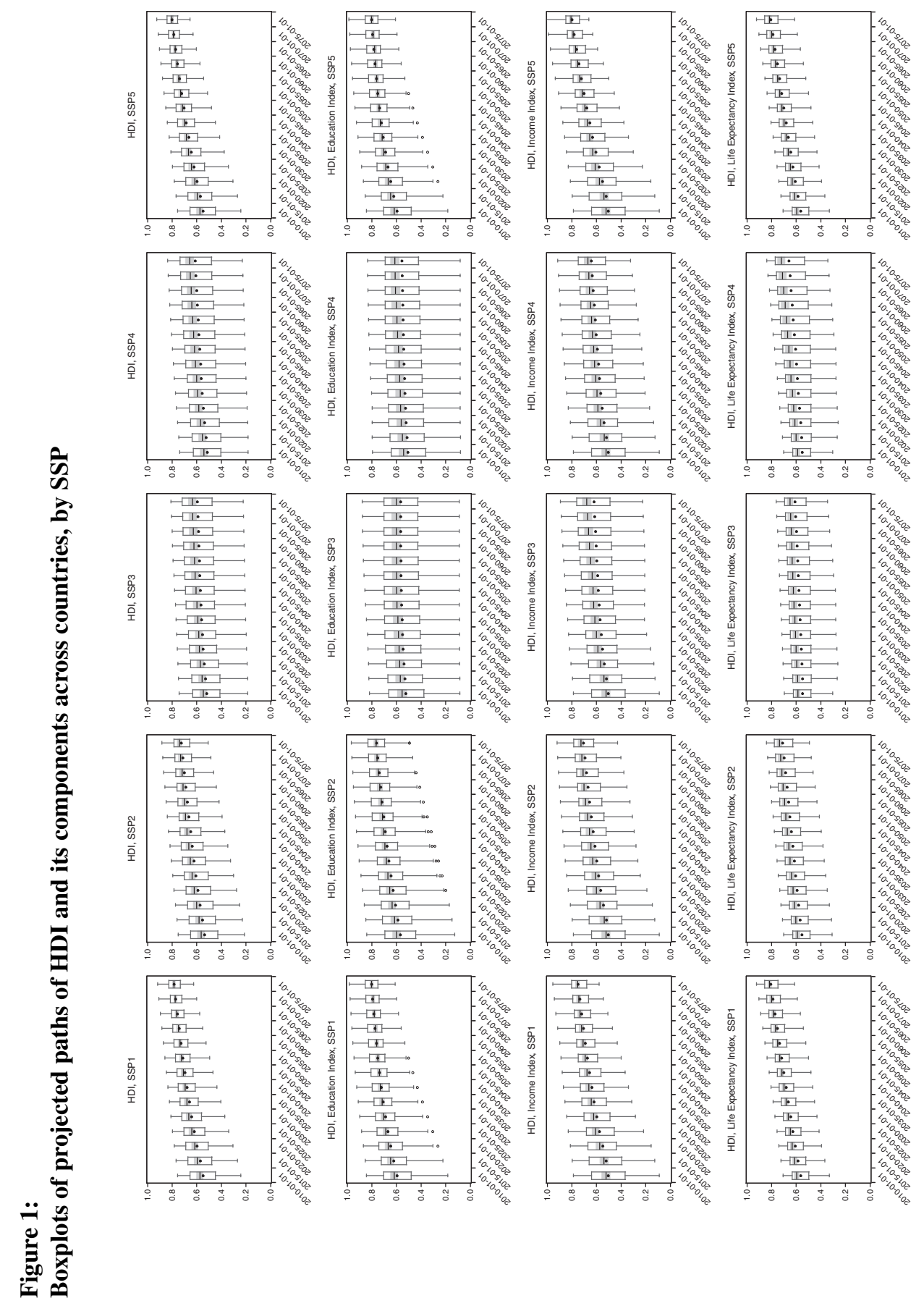


Figure 2:

HDI in 2010 against projected change between 2010 and 2075, by SSP

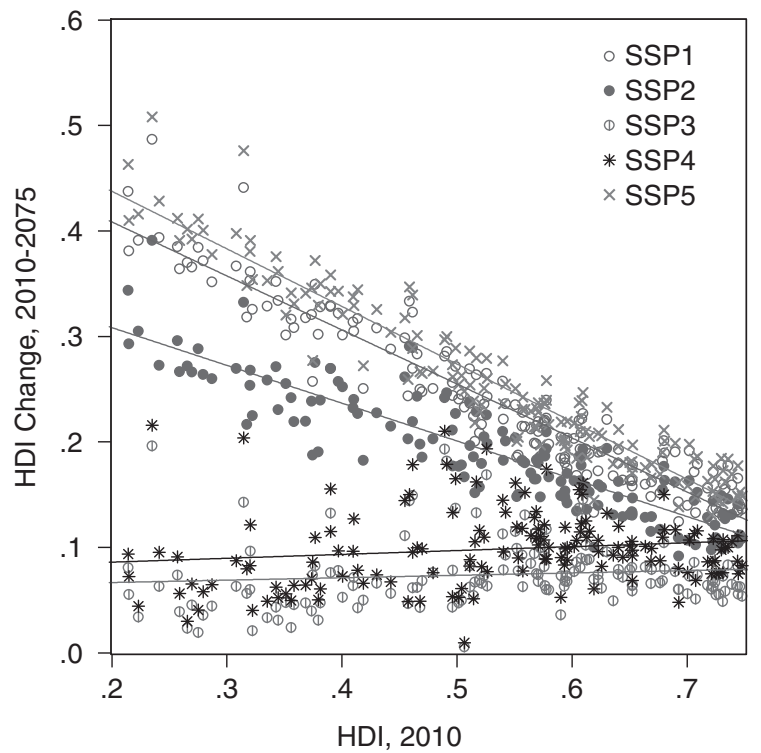

each of the sub-indices that compose the HDI. As expected, the pattern of crosscountry dispersion in the SSP2 scenario can be seen as being between the two patterns described: in SSP2 convergence occurs across economies, but there is a higher level of dispersion in the HDI than there is in SSP1 or SSP5.

The different convergence patterns in HDI projections can be better understood by plotting the HDI level in 2010 against the change in the level by country in the projection period 2010-2075. The scatterplot shown in Figure 2 reveals the different speeds of convergence across SSP scenarios. While in SSP1, SSP2, and SSP5 lower HDI values in 2010 tend to be strongly related to larger increases of the index in the period 2010-2075; in SSP3 and SSP4 no significant relationship is discernible between the initial HDI and the subsequent change over the projection period.

To illustrate the numerical results of these HDI projections exercise, Table 2 shows the trends under the five different scenarios for Ethiopia and Pakistan, two countries that are currently at the crossroads of either experiencing rapid development, or remaining stuck at a stalled level of development associated with low female education and high fertility (and thus high population growth). Both countries are currently classified as having low levels of human development, and rank 173 and 146, respectively, in the 2013 Human Development Report (United Nations Development Programme 2013). Since life expectancy is projected in fiveyear periods, the values for 2010 refer to the years 2010-2014, and already vary slightly by scenario. In each period of time, we base our measure of expected years of schooling on the mean years of schooling projected in 20 years for the age group 25-29. The differences in educational attainment dynamics across projection 
Table 2:

HDI projections by SSP: Ethiopia and Pakistan

\begin{tabular}{cccccc}
\hline \multicolumn{7}{c}{ Ethiopia } \\
\hline Year & SSP1 & SSP2 & SSP3 & SSP4 & SSP5 \\
\hline 2010 & 0.33 & 0.31 & 0.29 & 0.28 & 0.33 \\
2015 & 0.37 & 0.34 & 0.30 & 0.29 & 0.37 \\
2020 & 0.41 & 0.37 & 0.32 & 0.31 & 0.41 \\
2025 & 0.44 & 0.39 & 0.32 & 0.31 & 0.44 \\
2030 & 0.47 & 0.41 & 0.33 & 0.32 & 0.47 \\
2035 & 0.50 & 0.42 & 0.33 & 0.32 & 0.50 \\
2040 & 0.52 & 0.44 & 0.33 & 0.33 & 0.54 \\
2045 & 0.55 & 0.46 & 0.34 & 0.34 & 0.57 \\
2050 & 0.58 & 0.48 & 0.34 & 0.34 & 0.60 \\
2055 & 0.60 & 0.50 & 0.34 & 0.35 & 0.63 \\
2060 & 0.63 & 0.52 & 0.34 & 0.35 & 0.65 \\
2065 & 0.65 & 0.54 & 0.35 & 0.36 & 0.68 \\
2070 & 0.67 & 0.56 & 0.35 & 0.36 & 0.70 \\
2075 & 0.70 & 0.58 & 0.35 & 0.37 & 0.73 \\
\hline
\end{tabular}

Pakistan

\begin{tabular}{cccccc}
\hline Year & SSP1 & SSP2 & SSP3 & SSP4 & SSP5 \\
\hline 2010 & 0.42 & 0.40 & 0.37 & 0.36 & 0.42 \\
2015 & 0.45 & 0.42 & 0.37 & 0.37 & 0.45 \\
2020 & 0.47 & 0.44 & 0.38 & 0.37 & 0.48 \\
2025 & 0.50 & 0.46 & 0.38 & 0.38 & 0.50 \\
2030 & 0.53 & 0.48 & 0.39 & 0.38 & 0.53 \\
2035 & 0.55 & 0.50 & 0.39 & 0.39 & 0.56 \\
2040 & 0.58 & 0.52 & 0.39 & 0.39 & 0.59 \\
2045 & 0.61 & 0.54 & 0.40 & 0.40 & 0.62 \\
2050 & 0.63 & 0.56 & 0.40 & 0.41 & 0.64 \\
2055 & 0.65 & 0.58 & 0.41 & 0.41 & 0.67 \\
2060 & 0.67 & 0.60 & 0.41 & 0.42 & 0.69 \\
2065 & 0.69 & 0.62 & 0.41 & 0.42 & 0.71 \\
2070 & 0.71 & 0.64 & 0.42 & 0.43 & 0.73 \\
2075 & 0.73 & 0.65 & 0.42 & 0.43 & 0.75 \\
\hline
\end{tabular}

scenarios imply that this definition of expected years of schooling creates small differences in our HDI for the year 2010 as well. However, this strategy contributes to the internal consistency and the comparability of the projections across scenarios.

Viewed over time, the trends directly mirror the different narratives of the five SSPs. SSP1 assumes rapid progress over the coming decades. Under this scenario, Ethiopia would quickly catch up, and would even improve more rapidly than Pakistan (which starts from a somewhat higher level) under the same scenario. 
These trends can be attributed to the fact that Ethiopia has recently made considerable progress in expanding education (from a very low level) among the younger cohorts, whereas in Pakistan (which is at a somewhat higher level) less progress has been made. Thus, even under isomorphic assumptions, the future trends reflect current levels of momentum. On the other hand, under the stalled development scenario of SSP3 Ethiopia would see only very minor improvements in its development over the next 50 years. Over this period, the country would not even be able to catch up with Pakistan (Ethiopia would have an index of 0.35 in 2075, whereas Pakistan would have an index of 0.37 in 2010 under the same scenario).

The implications of such scenarios in terms of vulnerability to natural disasters related to climate change can be grasped by comparing the projected trajectories with the results presented in Patt et al. (2010). The analysis in Patt et al. (2010) suggests that climatic disaster risk (as measured by the number of persons affected by a climate disaster) is highest in the countries with HDI levels of around 0.5 , after controlling for other determinants of vulnerability. The number of affected individuals starts to decline only after countries reach this level of development. Using the elasticities obtained by Patt et al. (2010), we can compute the relative differences in the degree of vulnerability to climate disasters implied by each of our scenarios, keeping other determinants of vulnerability constant. The levels of development implied by SSP1 and SSP5 in 2075 imply that the ratio of individuals killed by climatic disasters to the total population will, on average, be approximately $90 \%$ and $118 \%$ higher, respectively, than in SSP2. Our projections for SSP3 and SSP4, on the other hand, indicate that the same variable will increase $150 \%$ to $175 \%$, respectively, relative to SSP2. As expected, the biggest reductions in vulnerability to climatic disasters will be made through improvements in human development (or, conversely, the largest increases in vulnerability will be associated with increases in cross-country inequality), which tend to be concentrated in the countries of SubSaharan Africa. These large differences in levels of vulnerability to natural disasters clearly show that development will play an important role in determining the impact of climate change on human populations.

\section{Conclusions}

To study the impact of climate change, we need to construct projection models in which the quantitative assessment of different socioeconomic paths at the global level plays a central role. As human development has been systematically found to be a key determinant of vulnerability to natural disasters, the construction of reliable projection models for education, health, and income indicators that span long time horizons is a key component of climate change assessment models.

We propose an internally consistent methodology for obtaining projections of the HDI and its components that can be used to address questions related to the future vulnerability of societies to climate change. The methodological framework rests on the combination of projections of population by age, sex, and educational attainment with a production function approach in which human capital dynamics (that is, the 
change in the composition of a population by age and educational attainment) is the main driving force of income changes over time.

Using the proposed methodology, we present HDI projections corresponding to the narratives of the five SSPs developed in the IPCC's Fifth Assessment Report. We show that the projection method is able to replicate the main global characteristics of these scenarios, and provides a new and useful quantitative instrument for climate change research. Furthermore, because this approach is self-contained, the methodology put forward in this study is useful for other applications that involve addressing the interactions between population, development, and the environment. Thus, the method complements existing quantitative approaches that assess the risks associated with climate change (see, for instance, Birkmann et al. 2013). Like all of the other global long-term projection methods, our approach is limited by the assumption that information about historical relationships can be used to help us understand the future dynamics of socioeconomic variables. But because structural breaks in these relationships could occur in the future, the particular assumptions underlying the different scenarios presented in this contribution need to be considered when using the projected paths of the HDI and its components.

\section{Acknowledgments}

The authors would like to thank the editors and two anonymous referees, as well as the participants of the international seminar Demographic Differential Vulnerability to Natural Disasters in the Context of Climate Change Adaptation, held in Kao Lak, for helpful comments on an earlier version of this piece.

\section{References}

Adger, W. N. 1999. Social vulnerability to climate change and extremes in coastal Vietnam. World Development 27: 249-269.

Barakat, B. F. and R. E. Durham 2014. Future education trends. In World population and human capital in the 21 st century, eds W. Lutz, W. P. Butz and S. KC. Oxford University Press.

Benhabib J. and M. Spiegel 1994. The role of human capital in economic development: Evidence from aggregate cross-country data. Journal of Monetary Economics 34: $143-173$.

Birkmann J., S. Cutter, D.S. Rothman, T. Welle, M. Garschagen, B. van Ruijven, B. C. O'Neill, B. Preston, S. Kienberger, O. D. Cardona, T. Siagian, D. Hidayati, N. Setiadi, C. Binder, B. Hughes and R. Pulwarty 2013. Scenarios for vulnerability: Opportunities and constraints in the context of climate change and disaster risk. Climate Change, 1-16.

Brooks, N., N. W. Adger and P. M. Kelly 2005. The determinants of vulnerability and adaptive capacity at the national level and the implications for adaptation. Global Environmental Change 15: 151-163.

Butz, W. P., W. Lutz and J. Sendzimir (eds) 2014. Education and differential vulnerability to natural disasters. Ecology $\mathcal{E}$ Society, Special Feature. 
Chakravarty, S. R. 2003. A generalized human development index. Review of Development Economics 7: 99-114.

Chakravarty, S. R. 2011. A reconsideration of the tradeoffs in the new human development index. Journal of Economic Inequality 9: 471-474.

Crespo Cuaresma, J. and T. Mishra 2011. The role of age-structured education data for economic growth forecasts. Journal of Forecasting 30: 249-267.

Crespo Cuaresma, J. 2015. Income projections for climate change research: A framework based on human capital dynamic. Global Environmental Change, in press, doi:10.1016/j.gloenvcha.2015.02.012.

Frankenberg, E., B. Sikoki, C. Sumantri, W. Suriastini and D. Thomas 2013. Education, vulnerability, and resilience after a natural disaster. Ecology $\mathcal{E}$ Society 18.

Garbero, A. and E. Pamuk 2014. Future mortality in high mortality countries. In World population and human capital in the 21 st century, eds W. Lutz, W. P. Butz and S. KC, pp. 273-332. Oxford: Oxford University Press.

Garbero, A. and W. C. Sanderson 2014. Appendix I: Forecasting mortality convergence up to 2100 . In World population and human capital in the 21 st century, eds W. Lutz, W. P. Butz and S. KC, pp. 650-665. Oxford: Oxford University Press.

IPCC 2013. Climate change 2013: The physical science basis. Contribution of Working Group I to the fifth assessment report of the Intergovernmental Panel on Climate Change, eds T. F. Stocker, D. Qin, G.-K. Plattner, M. Tignor, S. K. Allen, J. Boschung, A. Nauels, Y. Xia, V. Bex and P. M. Midgley, p. 1535. Cambridge University Press, Cambridge, United Kingdom and New York, NY, USA.

IPCC 2014. Climate change 2014: Synthesis report. Contribution of working groups I, II and III to the fifth assessment report of the intergovernmental panel on climate change, eds Core Writing Team, R. K. Pachauri and L. A. Meyer, p. 151. IPCC, Geneva, Switzerland.

KC, S. 2013. Community vulnerability to floods and landslides in Nepal. Ecology $\mathcal{F}$ Society 18(1): 8.

KC, S., M. Potančoková, R. Bauer, A. Goujon and E. Striessnig 2014. Data and methods. In World population and human capital in the 21st century, eds W. Lutz, W. P. Butz and S. KC, pp. 434-518. Oxford: Oxford University Press.

KC, S. and W. Lutz 2015. The human core of the SSPs: Population scenarios by age, sex and level of education for all countries to 2100. Global Environmental Change, in press, doi:10.1016/j.gloenvcha.2014.06.004.

Klugman, J., F. Rodriguez and H-J. Choi 2011. The HDI 2010: New controversies, old critiques. Journal of Economic Inequality 9: 249-288.

Klugman, J., F. Rodriguez and H-J. Choi 2011a. Response to Martin Ravallion. Journal of Economic Inequality 9: 497-499.

Kriegler, E., B. C. O’Neill, S. Hallegatte, T. Kram, R. Lempert, R. Moss and T. Wilbanks 2012. The need for and use of socio-economic scenarios for climate change analysis: A new approach based on shared socio-economic pathways. Global Environmental Change 22: $807-822$.

Lee, R. 2003. Mortality forecasts and linear life expectancy trends. In Social insurance studies 3: Perspectives on mortality forecasting, ed. T. Bengtsson. Swedish Social Insurance Agency. 
Lutz, W., A. Goujon, S. KC and W. Sanderson 2007. Reconstruction of populations by age, sex and level of educational attainment for 120 countries for 1970-2000. Vienna Yearbook of Population Research, 193-235.

Lutz, W., J. Crespo Cuaresma and W. Sanderson 2008. The demography of educational attainment and economic growth. Science 319: 1047-1048.

Lutz, W., W. P. Butz, M. Castro, P. Dasgupta, P. G. Demeny, I. Ehrlich, S. Giorguli, D. Habte, W. Haug, A. Hayes, M. Herrmann, L. Jiang, D. King, D. Kotte, M. Lees, P. K. MakinwaAdebusoye, G. McGranahan, V. Mishra, M. R. Montgomery, K. Riahi, S. Scherbov, X. Peng and B. Yeoh 2012. Demography's role in sustainable development. Science 335: 918-918.

Lutz, W., W. P. Butz and S. KC 2014. World population and human capital in the 21st century. Oxford: Oxford University Press.

Lutz, W. and S. KC 2012. Demography and human development: Education and population projections. UNDP-HDRO Occasional Papers No. 2013/04.

Muttarak, R. and W. Pothisiri 2013. The role of education on disaster preparedness: Case study of 2012 Indian Ocean earthquakes on Thailand's Andaman coast. Ecology $\mathcal{F}$ Society 18(4): 51.

Nakicenovic, N., J. Alcamo, A. Grubler, K. Riahi, R.A. Roehrl, H.-H. Rogner and N. Victor 2000. Special report on emissions scenarios (SRES), a special report of working group III of the intergovernmental panel on climate change, IPCC special reports on climate change. Cambridge University Press, Cambridge, UK.

Nelson R. and E. Phelps 1966. Investment in humans, technological diffusion and economic growth. American Economic Review, Papers and Proceedings 56: 69-75.

Patt, A. G., M. Tadross, P. Nussbaumer, K. Asante, M. Metzger, J. Rafael, A. Goujon and G. Brundrit 2010. Estimating least-developed countries' vulnerability to climate related extreme events over the next 50 years. Proceedings of the National Academy of Sciences of the United States of America 107: 1333-1337.

Pichler, A. and E. Striessnig 20103. Differential vulnerability to hurricanes in Cuba, Haiti, and the Dominican Republic: The contribution of education. Ecology E Society 18(3): 31.

Ravallion, M. 2010. Troubling tradeoffs in the human development index. World Bank Policy Research Working Paper 5485. The World Bank, Washington DC.

Ravallion, M. 2011. The human development index: A response to Klugman, Rodriguez and Choi. Journal of Economic Inequality 9: 475-478.

Striessnig, E., W. Lutz and A. Patt 2013. Effects of educational attainment on climate risk vulnerability. Ecology $\mathcal{E}$ Society 18(1): 16.

Torri, T. and J. W. Vaupel 2012. Forecasting life expectancy in an international context. International Journal of Forecasting 28: 519-531.

United Nations 2011. World population prospects: The 2010 revision. Department of Economic and Social Affairs, Population Division, New York.

United Nations Development Programme 2010. Human development report: The real wealth of nations. New York: Palgrave Macmillan.

United Nations Development Programme 2013. Human development report: The rise of the south: Human progress in a diverse world. New York: Palgrave Macmillan. 


\section{Appendix: Countries in the projection exercise}

\begin{tabular}{|c|c|c|}
\hline Algeria & Ghana & Pakistan \\
\hline Argentina & Greece & Panama \\
\hline Armenia & Guatemala & Paraguay \\
\hline Australia & Guinea & Peru \\
\hline Austria & Guinea-Bissau & Philippines \\
\hline Azerbaijan & Guyana & Poland \\
\hline Bahamas & Haiti & Portugal \\
\hline Bahrain & Honduras & Republic of Korea \\
\hline Bangladesh & Hong Kong & Romania \\
\hline Belarus & Hungary & Russian Federation \\
\hline Belgium & Iceland & Rwanda \\
\hline Belize & India & Saint Lucia \\
\hline Benin & Indonesia & Saint Vincent and the Grenadines \\
\hline Bhutan & Iran & Samoa \\
\hline Bolivia & Iraq & Sao Tome and Principe \\
\hline Bosnia and Herzegovina & Ireland & Saudi Arabia \\
\hline Brazil & Israel & Senegal \\
\hline Bulgaria & Italy & Serbia \\
\hline Burkina Faso & Jamaica & Sierra Leone \\
\hline Burundi & Japan & Singapore \\
\hline Cambodia & Jordan & Slovakia \\
\hline Cameroon & Kazakhstan & Slovenia \\
\hline Canada & Kenya & South Africa \\
\hline Cape Verde & Kuwait & Spain \\
\hline Central African Republic & Kyrgyzstan & Sudan \\
\hline Chad & Lao & Suriname \\
\hline Chile & Latvia & Swaziland \\
\hline China & Lebanon & Sweden \\
\hline Colombia & Lesotho & Switzerland \\
\hline Comoros & Liberia & Syria \\
\hline Congo & Lithuania & Tajikistan \\
\hline Costa Rica & Luxembourg & Tanzania \\
\hline Cote d'Ivoire & Madagascar & TFYR Macedonia \\
\hline Croatia & Malawi & Thailand \\
\hline Cuba & Malaysia & Timor-Leste \\
\hline Cyprus & Maldives & Tonga \\
\hline Czech Republic & Mali & Trinidad and Tobago \\
\hline Democratic Republic of the Congo & Malta & Tunisia \\
\hline Denmark & Mauritius & Turkey \\
\hline Dominican Republic & Mexico & Turkmenistan \\
\hline Ecuador & Moldova & Uganda \\
\hline Egypt & Mongolia & Ukraine \\
\hline El Salvador & Morocco & United Kingdom \\
\hline Equatorial Guinea & Mozambique & United States of America \\
\hline Estonia & Namibia & Uruguay \\
\hline Ethiopia & Nepal & Vanuatu \\
\hline Finland & Netherlands & Venezuela \\
\hline France & New Zealand & Viet Nam \\
\hline Gabon & Nicaragua & Zambia \\
\hline Gambia & Niger & Zimbabwe \\
\hline Georgia & Nigeria & \\
\hline Germany & Norway & \\
\hline
\end{tabular}


